The conception of entropy is vital in chemistry. The late Lord Rayleigh, in 1875 (or Horstman), laid the foundation of energeties by pointing out that all chemical actions went so as to increase entropy. I do not know which was first, neither did Lord Rayleigh.

19 Bramham Gardens, J. Swinburne.

London, S.W.5.

\section{Density of Crabs and Lobsters}

RECENTuY the densities of a large male crab, Cancer pagurus L., and a large male lobster, Homarus vulgaris Milne-Edwards, were found at Plymouth using the displacement method, and since-to use a popular phrase - these are the heaviest animals which move freely in sea-water the results may be of general interest. Both specimens were known to be in the late inter-moult stage. The density of the crab with a width of carapace $25 \mathrm{~cm}$. was found to be $1 \cdot 2784$ at a temperature of $8.0^{\circ} \mathrm{C}$., which gives a sinking factor of 1243. The density of the lobster $39.5 \mathrm{~cm}$. long was found to be 1.1871 at the same temperature, which gives a sinking factor of 1155. Thus the crab is considerably the denser.

The crab weighed practically $6 \frac{1}{2} \mathrm{lb}$., while the lobster weighed about $5 \frac{1}{2} \mathrm{lb}$. This is not by any means a record size for either animal, but both were fairly large specimens.

The technique for weighing these large animals is straightforward, but it requires care and preparation. Thus in this particular case it involved the procuring of an accurate balance capable of taking a load of 25 kilos, and a standard flask holding 20 litres. Finally, a density bottle had to be made and standardized, holding 10 litres.

Conditions are too precarious at present to think of carrying out a full investigation of the density of crabs and lobsters, since it would mean keeping a number of specimens of both sexes over a period of four or five years, but from the data already obtained it is possible to make the following generalizations :

(1) The density of the crab is greater than that of the lobster.

(2) The density of the male crab is definitely greater than that of the female.

(3) The density of a large crab is only very slightly greater than that of a small one. (The density of a male crab with a width of carapace of only $6 \cdot 8 \mathrm{~cm}$. was found to be $1 \cdot 2648$, which compares closely with the specimen given above with a width of carapace of $25 \mathrm{~cm}$. and a density of $1 \cdot 2784$.)

(4) In lobsters the density of the male seldom exceeds that of the female.

(5) The density of a large lobster is considerably greater than that of the smaller.

It is not difficult to account for the differences outlined above when it is remembered that in the lobster the size of the abdomen is comparable to that of the cephalothorax, while in the crab it is greatly reduced, and again a large part of the underside of the abdomen of the lobster is covered with thin chitin only. In the male lobster the claws are greatly enlarged but the abdomen is narrower. Considering the great increase in the size of the claws of the male crab it is not easy to understand the very slight increase in density, since one would look to heterogonic growth to cause a considerable increase.
'The Laboratory,
A. G. Lowndes.

\section{'White' Bread}

Mr. MASSINGHAM ${ }^{1}$ accuses millers and bakers of producing "faked" bread, basing his charge on the fact that pre-war flour was germ-free. He also states that eighteenth century white bread was never wheat-germless, and that at this period the process of "extraction" had not been invented.

Rich $^{2}$ has published figures showing the working of a typical flour mill that operated from 1786 until 1791. Far from "extraction" not having then been invented, "Fine Household Flour" consisted of $55 \cdot 3$ per cent only of the wheat berry, and from this flour was made the white bread then in general consumption in London. Another source from the same period gives 69 per cent for the 'extraction' of this type of flour.

It is probably true, however, that this flour was higher in vitamin $B_{1}$ than modern pre-war white flours, as Schultz, Atkin and Frey ${ }^{3}$ have found 1.5 r.U./gm. $B_{1}$ in 54-62 per cent extraction stoneground flours, made in as similar a manner as possible to the methods known to be in use a century ago.

The modern roller milling system is the result of much scientific research and produces the type of flour that, rightly or wrongly, people have striven for. When the War ends, the public in Great Britain will most probably demand white bread, as its appearance, eating and keeping properties, in their opinion, outweigh any nutritional advantages that our present bread may have.

The British Arkady Co., Ltd.,

A. M. Matden.

Skerton Road,

Old Trafford,

Manchester, 16.

${ }^{1}$ Nature, 5, 254 (1943).

J. Soc. Chem. Ind., 60, 612 (1941).

'Cer. Chem., 19, 529 (1942).

\section{Mechanical Lighters}

WheN about a year ago we found it difficult to get matches I thought of a plan for producing a light especially of value where gas is available, which if brought into use would mean economy in chlorate of potash and elimination of match-sticks and matchboxes-surely a desirable end. But then the definition of a mechanical lighter in the 1928 Act of Parliament was shown to me, which would impose a $2 s .6 d$. duty on an article which otherwise could be sold for a penny. I concluded, and the recent Bow Street Police Court tinder box case has shown that I was right, that the Excise would not hesitate to impose the duty on any lighter however advantageous it might be.

My plan was to make a paste of chlorate of potash with so much diluted silicate of soda as would, when dry, make a firm button which could be glued at the end of a rod and used to strike a spark against a safety match-box, or against a sheath coated with the same red phosphorus mixture. Hung up by a gas burner, it would light the gas with the expenditure of perhaps one tenth or one twentieth of the chlorate in a safety match head. To my surprise, I found it would light a methylated spirit lamp the only time I ever tried.

St. Marybourne,

C. V. Boys.

Plymouth. Jan. 24. Andover. 